\title{
The rotating visible outflow in M 82
}

\author{
A. Greve $e^{\star}$ \\ Institut de Radio Astronomie Millimétrique, 300 rue de la Piscine, 38406 St. Martin d’Hères, France \\ Received 21 March 2003 / Accepted 26 November 2003

\begin{abstract}
M 82's minor axis outflow is seen at visible wavelengths as more or less regular hollow cones on both sides of the galactic disk. The outflowing material is expected to entrain the rotation, or part of the rotation, of the disk where the outflow originates. From the conservation of angular momentum it is furthermore expected that the outflowing material continues to rotate at large distances from the disk, although probably with smaller velocity because of radial divergence of the cones. We provide evidence of this kinematic picture from long-slit spectra of the cone wall $\mathrm{H}_{\alpha}$, [NII] and [SII] emission lines taken at $\pm 20^{\prime \prime}( \pm 300 \mathrm{pc})$ and $\sim \pm 40^{\prime \prime}(\sim \pm 600 \mathrm{pc})$ distance from the center and parallel to the minor axis, from data extracted from the literature, and from a cone model fit of the data. The angular momentum which is entrained in the outflow and eventually dissipated is a small fraction of the total angular momentum associated with the stars and gas in the central part of the disk. We compare our observation of the visible outflow with the outflow of dragged-out material investigated in mm-wavelength $\mathrm{CO}$ by other observers. It seems that the material observed at visible wavelengths is confined to narrow cones, and blows out at velocities larger than the escape velocity of the galaxy. The dragged-out material moves at slower velocities and on wider cones, and may fall back into the galaxy.
\end{abstract}

Key words. galaxies: individual: M 82 - galaxies: structure - galaxies: ISM

\section{Introduction}

The supernova explosions and stellar winds of M 82's starburst produce an outflow of $10^{7}-10^{8} \mathrm{~K}$ hot gas along the minor axis on both sides of the galactic disk. This tenuous hot gas is collimated by the molecular gas ring and moves into the halo in the form of more or less regular cones. Because of M 82's favourable edge-on orientation (inclination $i \approx 80^{\circ}$, seen from below), the X-ray emission of the hot material inside the cones is seen to a distance of several kpc above and below the galactic plane (Bregman et al. 1995; Strickland et al. 1997). At the boundary between the hot gas and the disk and the halo, the outflow material shocks with swept-out material of the disk and with material of the halo, and cools to a temperature of $\sim 10^{4} \mathrm{~K}$. At this temperature the ionized gas $\left(n_{\mathrm{e}} \lesssim 500 \mathrm{~cm}^{-3}\right)$ emits the (forbidden) recombination lines of $\mathrm{H}_{\alpha}$, [NII], [SII]. This visible filamentary emission, confined to the cone walls, is seen to a height of 1-2 kpc on both sides of the disk with typical double velocity-split line profiles. The position-velocity analysis of the visible emission has revealed that the cones have opening angles of $\Omega \approx 20-30^{\circ}$ (full angle) (Burbidge et al. 1964; Axon \& Taylor 1978; Amirkhanyan et al. 1982, 1985; Bland \& Tully 1988 [BT-88]; Götz et al. 1990; McKeith et al. 1995 [M-95]; Shopbell \& Bland-Hawthorn 1998 [SBH-98]), although also considerably wider cones of $\Omega \approx 60^{\circ}$ opening angle have been proposed (Heckman et al. 1990 [HAM-90]). At the height of $\sim 10 \mathrm{kpc}$, at the NW side of the disk, the outflowing gas

\footnotetext{
* e-mail: greve@iram.fr
}

apparently bow-shocks with intergalactic gas and emits $\mathrm{H}_{\alpha}$ (Devine \& Bally 1999; Lehnert et al. 1999). Detection at visible wavelengths of rotation of cone wall material at large distances from the disk was mentioned by Bland \& Tully and Shopbell \& Bland-Hawthorn. Spectra taken by Heckathorn (1972) parallel to the major axis to a distance of 675 pc below and $410 \mathrm{pc}$ above the galactic plane have sloped tracks in the positionvelocity diagrams and by this clearly illustrate rotation of the visible outflow material, in the same direction as the material of the disk and with decreasing velocity for increasing distances from the disk. These and other imaging and spectroscopic observations were used to determine the geometry and kinematics of the visible outflow material; for a recent analysis and summary see SBH-98 and Table 1. Models of this type of starburst-driven outflow are published by Chevalier \& Clegg (1985), Tomisaka \& Ikeuchi (1988), Umemura et al. (1988), Heckman et al. (1990), Yokoo et al. (1993), Tenorio-Tagle \& Muñoz-Tuñon (1997), Strickland \& Stevens (2000), and others.

The observations indicate also that at the base, and probably mainly at the periphery of the visible cones, the outflow drags cool gas and dust out of the disk into the halo. At visible wavelengths $\left(\mathrm{H}_{\alpha}\right.$, [NII]) this dragged-out gas and dust is clearly seen in the images published by Ohyama et al. (2002 [OEA-02]). The dust in the halo is also seen to a height of $\pm 1-2 \mathrm{kpc}$ as scattered optical continuum emission of radiation apparently originating in the disk (BT-88), and by its optical polarization (Scarrott et al. 1991). Dragged-out dust is seen to a height of $\sim \pm 800 \mathrm{pc}$ in $1.2 \mathrm{~mm}, 0.85 \mathrm{~mm}$ and $0.45 \mathrm{~mm}$ 
continuum observations $^{1}$ (Alton et al. 1999; Thuma et al. 2000). Dragged-out molecular gas is seen in mm-wavelength $\mathrm{CO}$ emission to a height of approximately $0.5-1 \mathrm{kpc}$ above and below the disk (Nakai et al. 1987; Loiseau et al. 1990; Sofue et al. 1992). Loiseau et al. and Sofue et al. find that the molecular gas at the height between $0.5 \mathrm{kpc}$ and $1 \mathrm{kpc}$ rotates in the same direction as the disk, though with gradually smaller velocity at greater distances from the disk. The recent JCMT observation by Seaquist \& Clark (2001 [SC-01]) confirms without doubt $\mathrm{CO}$ material and its rotation up to a distance of $\sim 900 \mathrm{pc}$, on both sides of the disk. However, SC-01 find that the CO material moves out from the disk at a significantly smaller velocity than the visible cone wall material, that the $\mathrm{CO}$ material does not show velocity-split line profiles, and that the outflow of the molecular gas forms cones of a much wider opening angle of $\Omega \approx 40-90^{\circ}$. They argue also that the molecular material flows out under conservation of angular momentum, as already proposed by Nakai et al. (1987) and Sofue et al. (1992). Seaquist \& Clark conclude that the motion of the hot visible gas and of the cool dragged-out $\mathrm{CO}$ gas are distinctly different aspects of the outflow phenomenon. Further evidence of cool material being dragged-out in a cone-like form, at least at the SE side of the disk, comes from $\mathrm{CO}(1-0)$ observations made with the OVRO and IRAM 30 m telescopes by Walter et al. (2002 [WWS-02]). They find CO with $\sim 100 \mathrm{~km} \mathrm{~s}^{-1}$ velocity splitting at the SE side of the disk to a distance of $\sim 600 \mathrm{pc}$ below the plane, flowing out at the velocity of $230 \mathrm{~km} \mathrm{~s}^{-1}$ and forming a cone of $\sim 55^{\circ}$ opening angle. They do not find a similar cone-like outflow at the NW side of the disk. Their published position-velocity diagrams of $\mathrm{CO}$ at a distance of $350 \mathrm{pc}$ SE and NW from the disk show clearly rotation. Discussions on the energy and temperature of outflows and observational evidence of dragged-out material have led to the theory of mass-loaded outflows, as published by Tomisaka \& Bregman (1993), Suchkov et al. (1994, 1996), Hartquist et al. (1997), Strickland \& Stevens (2000), and others. For a sketch of these outflow phenomena see Fig. 1 in HAM-90, Fig. 5 in Alton et al. (1999), and especially Fig. 6 in OEA-02. In the terminology used by Ohyama et al. the cone walls are the ridge component, the dragged-out dust and gas component in the halo, outside the cones, is the diffuse component. The different spatial distributions are evident from their published pictures.

In this picture of a minor axis outflow the material of the visible cone walls is expected to rotate. We present new observations which trace in long-slit spectra parallel to the minor axis the rotation of the visible outflow material to a distance of $1-1.2 \mathrm{kpc}$ above and below the galactic plane.

In the observations, distances are originally measured in angular scale $(\theta)$. To be consistent with our earlier publication (M-95), we adopt that $1^{\prime \prime}$ is equivalent to $15 \mathrm{pc}$ at the distance of M 82 (3.1 Mpc; see Freedman et al. 1994 and Sakai \& Madore 1999 for a somewhat larger distance of 3.2-3.9 Mpc). The position angle of $\mathrm{M}$ 82's major axis is PA $\approx 60-65^{\circ}$, of the minor axis $\mathrm{PA} \approx 155^{\circ}$, as for instance extracted from the deep image published by Devine \& Bally (1999) but not

\footnotetext{
1 The broadband $1.2 \mathrm{~mm}$ and $0.85 \mathrm{~mm}$ observations contain a substantial amount of $\mathrm{CO}$ emission.
}

represented by the warped central CO distribution (Shen \& Lo 1995; Neininger et al. 1998). The Western side $(r<0)$ of M 82's disk moves towards the observer, the Eastern side $(0<r)$ moves away (Neininger et al. 1998; Greve et al. 2002): emission from the Western side is blue-shifted, from the Eastern side red-shifted. We note also that at visible wavelengths we do not necessarily observe the rotation of the disk core region (McKeith et al. 1993), which is hidden behind dust, but the rotation of material which lies mainly in front of the molecular ring of 600-800 pc diameter (Shen \& Lo 1995; Neininger et al. 1998; Weiß et al. 2001), or even further out. For this reason we do not discuss the optical emission inside $\sim \pm 200$ pc distance from the galactic plane.

\section{Optical observations}

Simultaneous observations of the $\mathrm{H}_{\alpha} 6563 \AA$, [NII] 6548, $6583 \AA$ and [SII] $6716,6731 \AA$ emission lines were made with the $2.5 \mathrm{~m}$ Isaac Newton Telescope (La Palma, Spain) at a spectral resolution of $0.84 \AA /$ pixel $=38 \mathrm{~km} \mathrm{~s}^{-1} /$ pixel (grating of 1200 grooves $/ \mathrm{mm}$ ) and a spatial resolution of $0.6^{\prime \prime} /$ pixel = $10 \mathrm{pc} / \mathrm{pixel}$. The seeing was $\sim 1^{\prime \prime}$, i.e. $\sim 15 \mathrm{pc}$. The slit of $3^{\prime}$ $(2.7 \mathrm{kpc})$ length was placed along the minor axis $\left(\mathrm{PA}=155^{\circ}\right)$, passing approximately through the center of M 82 defined by the $2.2 \mu \mathrm{m}_{\text {peak }}{ }^{2}$, and subsequently placed parallel to the minor axis at $\theta= \pm 20^{\prime \prime}(r= \pm 300 \mathrm{pc})$ and $\theta \approx \pm 40^{\prime \prime}(r \approx \pm 600 \mathrm{pc})$ radial distance from the center (in linear scale denoted by $r$ ). Because of the narrow cone opening and small base of the outflow, the on-axis spectrum and the $\pm 20^{\prime \prime}$ spectra show clear line splitting and allow the determination of the cone angle, the outflow velocity(ies) and acceleration, and the rotation; the $\pm 40^{\prime \prime}$ spectra are taken more or less along the cone edge where line splitting disappears and rotation is clearly observed.

The velocities of the emission components are extracted from 50 pc-binned spectra ( $z$-direction) by Gaussian profile fits; the accuracy of the velocity determination (peak of the Gaussian profile) is $\sim \pm 10 \mathrm{~km} \mathrm{~s}^{-1}(\sim 1 / 3$ pixel). The width of the lines $(F W H P)$ varies from $\sim 100 \mathrm{~km} \mathrm{~s}^{-1}$ to occasionally $200-250 \mathrm{~km} \mathrm{~s}^{-1}$, as also seen by other observers. We do not interpret the line widths of the fitted Gaussian profiles because of insufficient spectral resolution $(F W H P$ of skylines $\approx$ instrumental profile $\approx 1-1.5 \AA \leftrightarrow 50-70 \mathrm{~km} \mathrm{~s}^{-1}$ ). Confidence in the reality of faint emission features, and of the Gaussian component decomposition, is obtained from the similarity of the results deduced independently from the $\mathrm{H}_{\alpha}$, [NII] and [SII] lines.

The results are presented as position-velocity $[p-v]$ diagrams in which the systemic velocity $\left(200 \mathrm{~km} \mathrm{~s}^{-1}[ \pm 11]\right.$, Götz et al. 1990) has been subtracted. Figure 1a is the $p-v$ diagram at $-20^{\prime \prime}$ to the West $(r=-300 \mathrm{pc})$ of the center; Fig. $1 \mathrm{~b}$ the $p-v$ diagram at $20^{\prime \prime}$ to the East $(r=300 \mathrm{pc})$ of the center. Line splitting is observed, although a very regular flow pattern is at some places absent. Figure 1 shows as reference the $p-v$ diagram along the minor axis $(r=0)$ obtained in this observation and the earlier observation by M-95.

Figure 2 shows the $p-v$ diagram parallel to the minor axis at $\theta \approx \pm 40^{\prime \prime}(r \approx \pm 600 \mathrm{pc}$ ) distance from the center. Emission

\footnotetext{
${ }^{2}$ RA 09 $51^{\mathrm{m}} 43.5^{\mathrm{s}}, \operatorname{Dec} 69^{\circ} 55^{\prime} 0.7^{\prime \prime}$ [1950] (Dietz et al. 1986).
} 


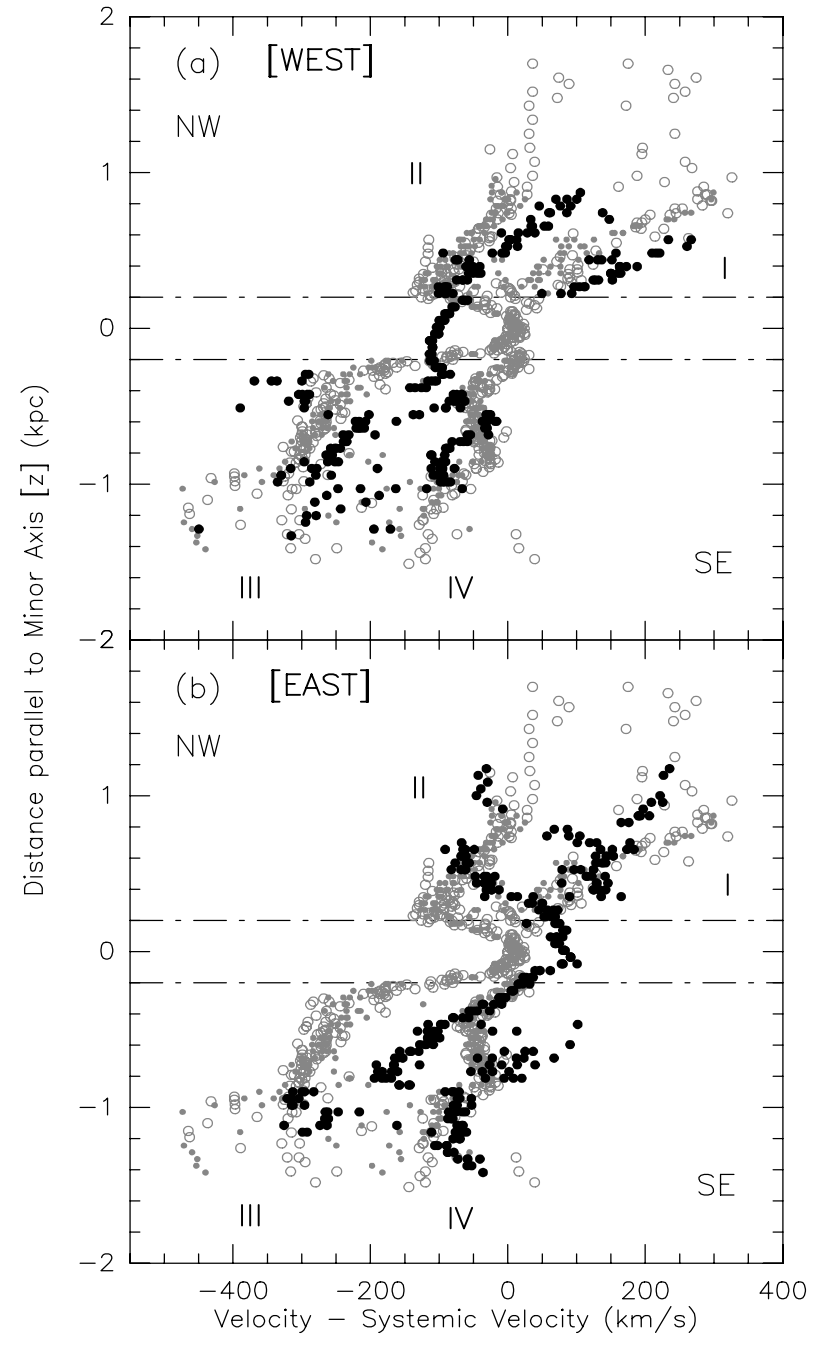

Fig. 1. a) BLUE-shifted outflow (solid dots) measured parallel to the minor axis at $\theta=-20^{\prime \prime}(r=-300 \mathrm{pc})$ to the West of the center. The gray symbols (open circles: M-95) represent the outflow along the minor axis $(r=0)$. The dashed-dotted lines indicate a galactic disk of $\pm 200 \mathrm{pc}$ thickness. The projected rotation of the disk is $v(-300) \approx$ $-100 \mathrm{~km} \mathrm{~s}^{-1}$, as seen at visible wavelengths. b) RED-shifted outflow (solid dots) measured parallel to the minor axis at $\theta=20^{\prime \prime}$ $(r=300 \mathrm{pc})$ to the East of the center. The projected rotation of the disk is $v(300) \approx 85 \mathrm{~km} \mathrm{~s}^{-1}$, as seen at visible wavelengths.

of the cone walls is still visible at this distance from the center (contrary to a very thin cone for instance shown in Fig. 9 by Strickland \& Stevens 2000) and the emission extends continuously to a height of $\sim \pm 1 \mathrm{kpc}$ from the disk. This leads us to conclude that the width of the outflow is $\Delta b \approx 150-250 \mathrm{pc}$. At the SE side of the disk we do not observe line splitting which confirms that we observe the edge, or regions close to the edge, of the visible cone. The outflow at the NW side of the disk observed to the East from the center $\left(\theta \approx 40^{\prime \prime}\right)$ shows some velocity splitting. This gives the impression that the NW cone is tilted in the plane perpendicular to the line-of-sight in the direction East, as already mentioned by Lynds \& Sandage (1963) and also seen in the image taken by Deharveng \& Pellet (1970). However, our observation is not sufficient to quantify this tilt. The literature contains a spectrogram of the $\mathrm{H}_{\alpha}$ line taken with

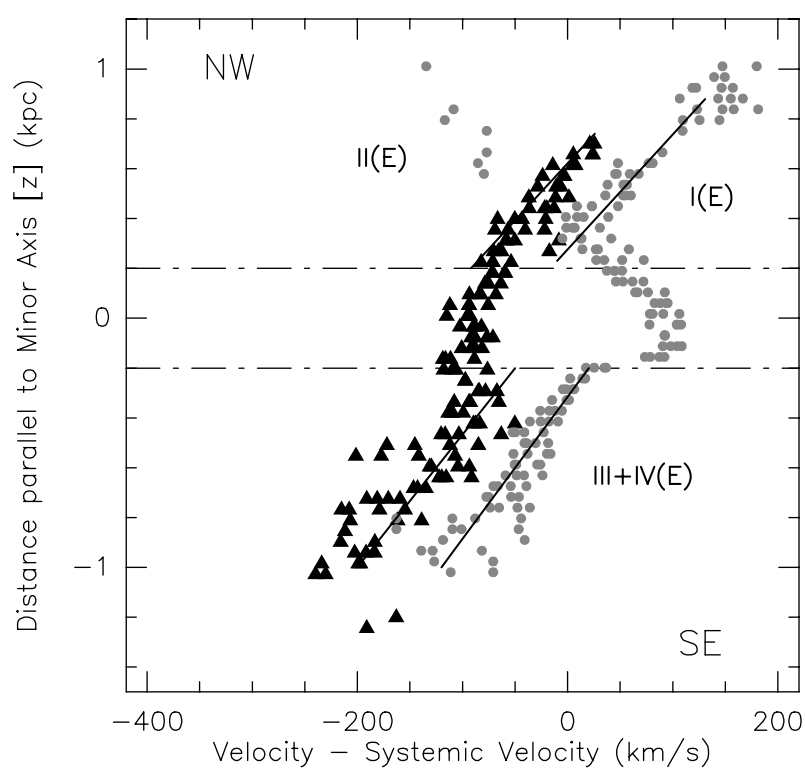

Fig. 2. Red-shifted outflow (dots) measured parallel to the minor axis at $\theta \approx 40^{\prime \prime}(r \approx 600 \mathrm{pc})$ to the East of the center, and Blue-shifted outflow (triangles) measured at $\theta \approx-40^{\prime \prime}(r \approx-600 \mathrm{pc})$ to the West of the center. The dashed-dotted lines indicate a galactic disk of $\pm 200 \mathrm{pc}$ thickness. The projected rotation velocity of the disk is $v( \pm 600) \approx$ $\pm 100 \mathrm{~km} \mathrm{~s}^{-1}$. The velocity difference between the Western and Eastern branch at the SE and NW side of the disk can be taken as evidence of rotation. For the straight lines on the SE and NW side see Sect. 6.4.

the slit parallel to the Northern part of the minor axis at $\sim 50^{\prime \prime}$ to the East of the center (Devine \& Bally 1999, their Fig. 5). This spectrogram shows a velocity splitting of $\sim 200-300 \mathrm{~km} \mathrm{~s}^{-1}$, up to the distance of $\sim 1 \mathrm{kpc}$ or more. Some of this velocity-split emission is probably seen in our observation at a similar velocity separation (Fig. 2).

Figures 1 and 2 show that the outflow breaks out of the disk at a distance of $\sim \pm 200 \mathrm{pc}$ from the galactic plane. Line splitting is seen above this height and can be traced to a distance of $\sim 1-1.5 \mathrm{kpc}$ on both sides of the galactic plane.

\section{Cone or bubble?}

In the discussion of superwind outflows we may distinguish between a cone-like geometry or a bubble-like geometry. Using a simple description, a cone-like outflow has a broad base in the disk, no top, and is open; line splitting is seen up to the top (for instance M 82, NGC 253). A bubble-like outflow has a small base in the disk, a top, and is closed, at least partially; line splitting disappears at the top (for instance NGC 3097, NGC 1482). Our observations, and in particular the observation along or close to the minor axis shown in Figs. 1 and 3, give some indication of emission at a distance of $\sim 1-1.5 \mathrm{kpc}$ at the SE side of the disk which fills the gap between the velocitysplit branches III-IV (for the branches see Fig. 4). These observations give the impression of a bubble, at least at the SE side of the disk. The closed velocity structure was noticed by BT- 88 on the SE and NW side at $\sim 900$ pc distance from the disk; it is also indicated in the observation by HAM-90 (their Fig. 10) where the SE and NW cones seem to be closed at the 


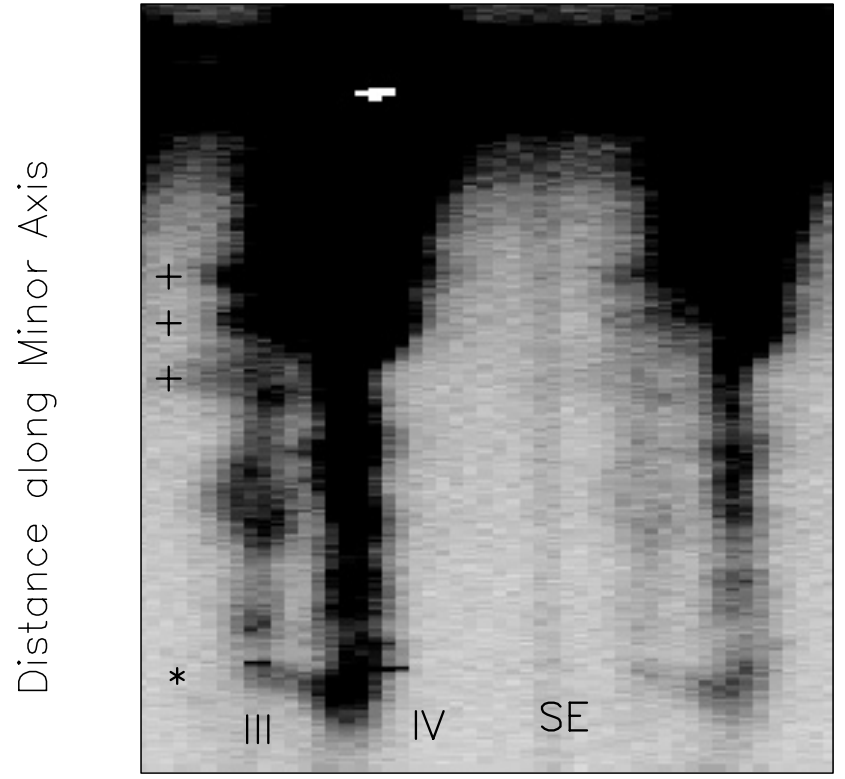

Fig. 3. $\mathrm{H}_{\alpha} 6563 \AA$ (left) and [NII] $6583 \AA$ (right) emission lines observed (4.2 m WHT) parallel and close to the minor axis $\left(|\theta| \leq 5^{\prime \prime}\right)$. The spatial direction is vertical, NW is at the top, SE at the bottom; the wavelength (velocity) direction is horizontal. The profiles show on the SE side at $z \approx-1 \mathrm{kpc}$ distance some emission between the velocitysplit cone sections $(*)$. Note the breakout from cone wall III (SE side, blue-shifted) close to the galactic disk (+).

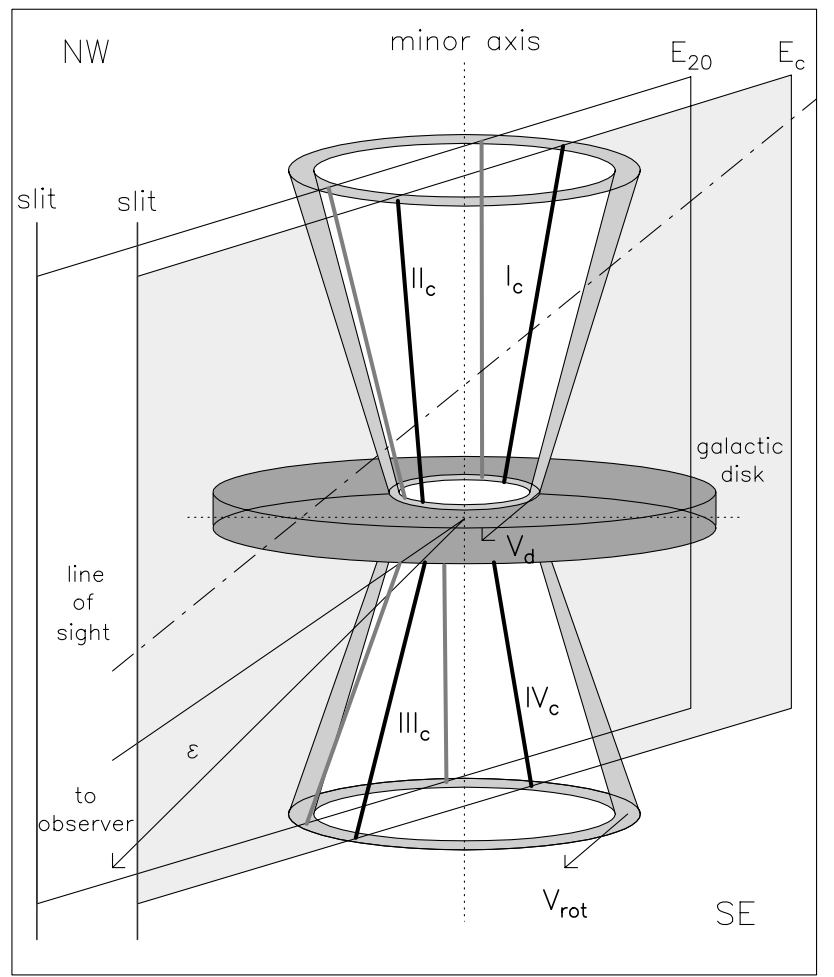

Fig. 4. Illustration of an idealized outflow cone on the SE and NW side of the galactic disk. $\mathrm{E}_{\mathrm{c}}$ : plane of the slit along the minor axis through the center of the galaxy, producing the velocity branches (I, II, III, IV) $)_{\mathrm{c}}$ (on-axis profile in Fig. 1); $\mathrm{E}_{20}$ : plane of the slit parallel to the minor axis at $\theta=20^{\prime \prime}$ to the East of the center, producing the velocity branches (I, II, III, IV) 20 (Fig. 1b) (similar for $\theta=-20^{\prime \prime}$, West). The rotation of the disk $v_{\mathrm{d}}$ and of the halo material $V_{\text {rot }}$ is shown. $\epsilon=90^{\circ}-i$ is the tilt of the galaxy. For a similar schematic see HAM-90. top. A similar impression of closure, or partial closure, of the SE cone at a distance of $\sim 2 \mathrm{kpc}$ below the disk is obtained from the optical image shown by Deharveng \& Pellet (1970) and the Fabry-Perot images published by SBH-98. The $\mathrm{H}_{\alpha}$ image published by OEA-02 gives at the NW side of the disk the impression of an outflow of diffuse material with some bubblelike structure extending to $\sim 2.5 \mathrm{kpc}$ distance from the disk, and on the SE side of the disk the impression of more or less cylindrical cones (as earlier mentioned by Lynds \& Sandage 1963). The CO observation by WWS-02 shows at the SE side of the disk an outflow cone and at $\sim 660 \mathrm{pc}$ distance from the disk emission which fills the velocity branches (however, at this position there is confusion with a sidelobe in the interferometer observation; Weiß, priv. comm.). The closure seems also to be indicated in the X-ray observations. Bregman et al. (1995) note that the X-ray cone at the SE side of the disk shows a significant change from a wide cone angle $\left(\Omega \approx 60^{\circ}\right)$ to a narrow cone angle $\left(\Omega \approx 20^{\circ}\right)$ at the distance of $\sim 1.7 \mathrm{kpc}$.

We adopt, despite this apparently bubble-like velocity feature, a cone-like outflow geometry, also since there exists collimated emission at a larger distance than the apparent closure. In addition to these observations, we note that according to SBH-98 there exists no acceptable model of a bubble which fits the observations, at least at the SE side of the disk.

\section{Parameters of the outflow cones}

\subsection{Geometry and kinematics of the outflow}

An idealized picture of the outflow cones, and of the selected slit positions, is shown in Fig. 4. The geometry of the outflow

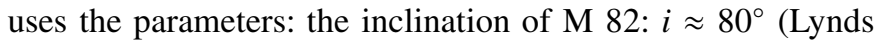
$\&$ Sandage 1963); the direction of the inclination: the northern side of the galaxy is tipped away from the observer; the distance $r \equiv r(z=0)$ measured along the major axis $(r<0$ : West, $0<r$ : East) and parallel to the major axis, $r(z)$, at the distance $z$ from the disk; the distance $z$ above and below the galactic plane $(0<z$ : direction NW, $z<0$ : direction SE), and $Z=|z|$; the thickness of the galactic disk: $2 H(0<H \approx 200 \mathrm{pc})$; the diameter at the base of the cone: $B=2 b$; the thickness of the cone wall: $\Delta b$; the full opening angle of the SE cone and NW cone: $\Omega_{\mathrm{SE}}$ and $\Omega_{\mathrm{NW}}$; the tilt of the SE cone and NW cone in the direction of the line-of-sight: $\Theta_{\mathrm{SE}}$ and $\Theta_{\mathrm{NW}}$; and the tilt in the plane perpendicular to the line-of-sight: $\Psi_{\mathrm{SE}}$ and $\Psi_{\mathrm{NW}}$. We do not distinguish between the true distance $\left(z^{*}\right)$ and the projected distance $(z)$ along the minor axis since $z^{*}=z / \sin (i) \approx 1.04 z \approx z$.

The kinematics of the outflow uses the parameters: the distance measured along the cone walls: $\rho$; the true (de-projected) blowout velocities at the base of the cone and at the edge of the disk (at $z= \pm H \approx \pm 200 \mathrm{pc}$ ): $V_{\mathrm{b}, \mathrm{SE}}$ and $V_{\mathrm{b}, \mathrm{NW}}$; the true gradient (increase) of the outflow velocity along the cone wall: $\partial V_{\mathrm{SE}}(\rho) / \partial \rho=a_{\mathrm{SE}}(\rho)$ and $\partial V_{\mathrm{NW}}(\rho) / \partial \rho=a_{\mathrm{NW}}(\rho)$; and the tangential component of the rotation of cone wall gas: $V_{\text {rot }}(r, z)$ at the distance $r$ from the minor axis and $z$ from the disk. The tangential component of the rotation of disk material is $v(r)$. Only the component of a velocity perpendicular to the plane of the sky can be measured. 
For the true outflow velocity along the cone walls (distance $\rho$ ) at the SE and NW side of the disk we use

$V(\rho)=V_{\mathrm{b}}+a(\rho) \times \rho$

with $\rho=0$ at the edge of the disk at $z \approx \pm 200 \mathrm{pc}$. The blowout velocity $V_{\mathrm{b}}$ and the acceleration $a(\rho)$ may be different at the SE and NW side of the disk. Following the notation of M-95 (their Figs. 2, 5), the projected velocity components of the front side and rear side of the SE cone are $V_{\mathrm{III}}$ and $V_{\mathrm{IV}}$, respectively, and equivalent $V_{\mathrm{II}}$ and $V_{\mathrm{I}}$ for the NW cone. Using $\epsilon=90^{\circ}-i$, $\Psi_{\mathrm{SE}}=\Psi_{\mathrm{NW}} \approx 0$ because of lack of observational information, and $\Theta_{\mathrm{SE}}$ and $\Theta_{\mathrm{NW}}$ counted positive if the respective cone is tilted towards the observer, the projected velocities measured with the slit centered along the minor axis are for the SE cone

$V_{\mathrm{III}, \mathrm{IV}}=\mp V_{\mathrm{SE}}(\rho) \sin \left(\Omega_{\mathrm{SE}} / 2 \pm \epsilon \pm \Theta_{\mathrm{SE}}\right)$

with the upper sign for branch III, the lower sign for branch IV. For the NW cone

$V_{\mathrm{II}, \mathrm{I}}=\mp V_{\mathrm{NW}}(\rho) \sin \left(\Omega_{\mathrm{NM}} / 2 \mp \epsilon \pm \Theta_{\mathrm{NW}}\right)$

with the upper sign for branch II, the lower sign for branch I. At a selected height $Z=|z|$, the distances $\rho$ in Eqs. (2) and (3) where the line-of-sight intersects the walls of the SE (III, IV) cone and the NW (I, II) cone, are

$\rho_{\mathrm{III}, \mathrm{IV}}=\left[Z+D_{\mathrm{III}, \mathrm{IV}}\right] / \cos \left(\Omega_{\mathrm{SE}} / 2 \pm \epsilon \pm \Theta_{\mathrm{SE}}\right)$

$\rho_{\mathrm{II}, \mathrm{I}}=\left[Z+D_{\mathrm{II}, \mathrm{I}}\right] / \cos \left(\Omega_{\mathrm{NW}} / 2 \mp \epsilon \pm \Theta_{\mathrm{NW}}\right)$

with

$D_{(\mathrm{III}, \mathrm{I}),(\mathrm{IV}, \mathrm{II})}=D_{\mathrm{I}}=-H / \cos \epsilon \pm b \sin \epsilon$.

The projected velocities Eqs. (2) and (3) measured along the minor axis do not reveal rotation; for this slit position a possibly entrained galactic rotation is expected to be perpendicular to the line-of-sight and thus not visible. Primarily spectra taken along the minor axis were used by HAM-90, M-95 and SBH-98 to derive the geometry of the outflow.

Figure 4 shows also the situation of a slit parallel to the minor axis $(\theta \neq 0)$. At the base of the outflow the radius of the cone is $b^{*}$, at the height $Z=|z|$ its radius is

$R(z)=b^{*}+(Z-H) \tan (\Omega / 2) / \sin (i), \quad H \leq Z$

with $\Omega$ either $\Omega_{\mathrm{SE}}$ or $\Omega_{\mathrm{NW}}$, and $H=200 \mathrm{pc}$. For idealized cones, Eq. (7) holds throughout the thickness of the cone wall, i.e. for material inside $b-\Delta b / 2 \leq b^{*} \leq b+\Delta b / 2$, where $\Delta b$ is of the order of 150-200 pc (Table 1).

We assume that the rotation $v(r)=V_{\text {rot }}(r, z=0)$ of the disk at the off-center position $r= \pm b^{*}$, or a part of this rotation, is entrained by the outflow. We assume also that the visible material (ridge material) flows out primarily along the cone walls, with little diffusion in perpendicular radial direction (see Fig. 3). If the rotation $V_{\text {rot }}(r, z)$ at the distance $r(z)$ from the minor axis and distance $z$ from the disk is known, then the lineof-sight velocity $\boldsymbol{V}_{\text {obs }}$ measured at the off-center slit position $( \pm|r|)$ is

$$
\begin{aligned}
\boldsymbol{V}_{\text {obs }}(r, z, i) & =\boldsymbol{V}_{\text {out }}(i)+\boldsymbol{V}_{\text {rot }} \\
& =V_{\mathrm{i}} \sin [\varphi(z)] \pm V_{\text {rot }}(r, z) \cos [\varphi(z)] \cos (\epsilon)
\end{aligned}
$$

with

$\sin [\varphi(z)]=\sqrt{R(z)^{2}-r^{2}} / R(z), \quad \cos [\varphi(z)]=r / R(z)$

and $i=\mathrm{I}$, II, III, IV for the velocity branch under discussion. The minus sign in Eq. (8) refers to the Western side (blue-shifted) of the center, the plus sign to the Eastern side (red-shifted) of the center. The relation (8) shows that the observed velocity $\boldsymbol{V}_{\text {obs }}$ consists of the components $\boldsymbol{V}_{\text {out }}$ and $\boldsymbol{V}_{\text {rot }}$. In order to derive the rotation $\boldsymbol{V}_{\text {rot }}$ it is convenient to eliminate the outflow component $\boldsymbol{V}_{\text {out }}$ from the observation. This can for instance be done by taking the difference of the velocities $\boldsymbol{V}_{\text {out }}(r, z)$ and $\boldsymbol{V}_{\text {out }}(-r, z)$ observed at opposite sides of the cone, or at the edges.

\subsection{Cone opening angle, diameter of the base, cone wall thickness}

Table 1 reviews the geometry of the cones as determined in and from earlier optical investigations and the recent radio observations. Some of the earlier observers (until 1990) assumed that the outflow originates from a small volume at the center of the galaxy so that the cone-like outflow has a vertex (called "pointed" in Table 1). From Table 1 we adopt, for the time being, for the visible outflow cones on both sides of the disk that $\Omega=20-30^{\circ}, B=400-800 \mathrm{pc}$, and $\Delta b=150-200 \mathrm{pc}$. We extract from our on-axis observation $(\theta=0)$ primarily the geometry $(\Omega, b)$, the outflow velocity $\left(V_{\mathrm{b}}\right)$, and the acceleration $(a)$. These values are used in the off-axis observations $\left(\theta= \pm 20^{\prime \prime}\right)$ to investigate the rotation of the outflow.

\subsection{Angular momentum conservation}

A collision-free outflow conserves mass and angular momentum. The combination of both laws of conservation provides the theoretical rotation velocity $\boldsymbol{V}_{\text {rot }}(r, z)$ as function of radial distance $(r)$ from the minor axis and distance $(z)$ from the disk, to be used in Eq. (8). As illustrated in Fig. 5, we assume that the mass $m_{\mathrm{cl}}$ of an individual cloud (filament, particle) does not change and that its angular momentum $\boldsymbol{L}$ is conserved while the material follows a trajectory along the gradually increasing radius $R(z)$ of the cone walls. The relevant relations are

$\boldsymbol{L}=m_{\mathrm{cl}} R(z) \boldsymbol{V}_{\text {rot }}(R, z)=$ constant

$\boldsymbol{V}_{\text {rot }}(R, z) R(z)=$ constant

since $m_{\mathrm{cl}}=$ constant. The distance $R(z)$ as function of distance $z$ is given by Eq. (7). We use the angular momentum $\boldsymbol{L}_{\mathrm{vis}}$ for visible cone wall material with $b=300-600 \mathrm{pc}, \Omega=25^{\circ}$, and the angular momentum $\boldsymbol{L}_{\mathrm{do}}$ for dragged-out material with $b=300 \mathrm{pc}, \Omega=50^{\circ}$. Evidently, at the base of the outflow $\boldsymbol{L}_{\mathrm{vis}}(b, \pm 200)=m_{\mathrm{cl}} b^{*} v\left(b^{*}\right)$, and similarly for $\boldsymbol{L}_{\mathrm{do}}$.

\section{The adopted model of the outflow}

We agree with the other investigators that the visible outflow at the SE side has to a large extent the geometry of a cone; however, contrary to SBH-98 and in agreement with our earlier observation (M-95) we find that the outflow at the NW side is also 
Table 1. Cone opening angle(s) $\Omega$, diameter of the outflow base $B=2 b$, and cone wall thickness $\Delta b$ determined in earlier optical observations and recent radio observations.

\begin{tabular}{|c|c|c|c|}
\hline Observation & $\begin{array}{c}\text { Cone angle } \Omega \\
{\left[{ }^{\circ}\right]}\end{array}$ & $\begin{array}{c}\text { Diameter } \\
\text { at Base } B[\mathrm{pc}]\end{array}$ & $\begin{array}{c}\text { Cone Wall } \\
\text { Thickness } \Delta b[\mathrm{pc}]\end{array}$ \\
\hline \multicolumn{4}{|l|}{ Optical Observation } \\
\hline (1) Lynds \& Sandage (1963) & "closed loops" $(\Omega=120)$ & & \\
\hline (2) Burbidge et al. (1964) & $\Omega \approx 20$ & pointed & \\
\hline (3) Deharveng \& Pellet (1970) & "loop" & & $30 ; 300$ \\
\hline (4) Heckathorn (1972) & cone & pointed & \\
\hline (5) Axon \& Taylor (1978) & cone & & \\
\hline (6) Bland \& Tully (1988) & "bubbles" & pointed & \\
\hline (7) Heckman et al. (1990) & $\Omega_{\mathrm{SE}}=\Omega_{\mathrm{NW}}=62$ & pointed & \\
\hline (8) Götz et al. (1990) & $\Omega=30$ & $B_{\mathrm{o}}=750$ & \\
\hline (9) McKeith et al. (1995) & $\Omega=30$ & $\sim 500$ & \\
\hline (10) Shopbell et al. (1998) & SE: $\Omega_{\mathrm{i}}=5 ; \Omega_{\mathrm{o}}=25$ & $375-400$ & \\
\hline \multicolumn{4}{|l|}{ Derived by us from: } \\
\hline (11) Width Ellips (BT-88) & & & 250 \\
\hline (12) Width Ellips (HAM-90) & & & $200-400$ \\
\hline (13) Thickness Filaments (DB-99) & & & $150-200$ \\
\hline \multicolumn{4}{|l|}{ Radio Observation (CO) } \\
\hline (14) Seaquist \& Clark (2001) & $\Omega_{\mathrm{SE}}=90, \Omega_{\mathrm{NW}}=40$ & $\sim 750$ & $\sim 300$ \\
\hline (15) Walter et al. (2002) & $\Omega_{\mathrm{SE}}=55$ & $\sim 400$ & $\sim 250$ \\
\hline \multicolumn{4}{|l|}{ Derived by us from: } \\
\hline (16) Neininger et al. (1998) & & $B_{\mathrm{i}} \approx 300 ; B_{\mathrm{o}} \approx 800$ & $\sim 250$ \\
\hline
\end{tabular}

SE: South-Eastern side of disk; NW: North-Western side of disk.

Remarks made by the authors or by us: (1) LS.: opening angle used in a model discussion. (2) B. et al.: optically thick cone, only the front is visible. (3) DP.: apparent width of filament $\sim 2^{\prime \prime}=30 \mathrm{pc}$; (us): thickness of cone limbs (300 pc).

(5)-(10): hollow cones are mentioned.

(6) BT.: SE bubble is cone-like up to $z=-975 \mathrm{pc}, \Omega=35^{\circ}$. (7) H. et al.: pointed, their Fig. 19; tilted by $\Theta \approx 30-40^{\circ}$.

(8) G. et al: measured at $z=150$ pc parallel to major axis; at smaller distances no line splitting.

(10) SBH.: $\Omega_{\mathrm{i}}$ : inner cone, $z \lesssim-350 \mathrm{pc} ; \Omega_{\mathrm{o}}$ : outer cone.

(11) (us): at $z=-750 \mathrm{pc}$ parallel to major axis.

(12) (us): at $z=-900 \mathrm{pc}$ parallel to major axis.

(13) (us): derived from the image published by Devine \& Bally.

$(14)+(15)$ (us): $B$ and $\Delta b$ extracted from published angular momentum diagrams.

(16) (us): $B_{\mathrm{i}}$ and $B_{\mathrm{o}}$ are the CO ring inner and outer diameter; $\Delta b=\left(B_{\mathrm{o}}-B_{\mathrm{i}}\right) / 2$. Similar values follow from the data

published by Telesco et al. (1991), Achtermann \& Lacy (1995), Weiß et al. (2001), and Mao et al. (2000).

cone-like. The parameters of the adopted best-fit cone model as derived from the presented observation are summarized in Table 2; the correspondingly calculated velocity branches I-IV for the slit along the minor axis, or parallel to the minor axis $\left(\theta= \pm 20^{\prime \prime}\right)$, are shown in Fig. 6 .

In general we obtain an acceptable fit of the observations if we adopt for M 82's inclinitation the value $i=75^{\circ}$, instead of $i=81^{\circ}$ derived by Lynds \& Sandage (1963). We note that the inclination of the velocity branches I-IV in the respective $p-v$ diagrams can only be explained by accelerated motion of the outflow; the values are given in Table 2. It is also important to note that a fit of the NW cone is only obtained if we introduce an additional line-of-sight velocity shift of $-100 \mathrm{~km} \mathrm{~s}^{-1}$ towards the observer, as given in Table 2 and illustrated in Fig. 6 (top panel). We have searched for an explanation other than a velocity shift by varying the base of the outflow, the cone angle, the blowout velocity, and the tilt of the cone, however, without finding an acceptable solution. For the time being we accept this velocity shift, knowing that further confirmation is required.

\section{Rotation above the disk}

\subsection{Rotation of the disk}

Any rotation entrained by the visible outflow material, or the dragged-out material, originates from the rotation of disk material. Since the visible outflow is seen to at least a distance of $\pm 600 \mathrm{pc} \lesssim r$ from the center (Fig. 2), we are concerned with rotation of disk material inside this region. For the rotation of the disk material we use the rotation curves published by McKeith et al. (1993) and Greve et al. (2002). We have shown (Greve et al. 2002) that M 82 contains a stellar bar within $r \lesssim \pm 30^{\prime \prime}(\sim \pm 500 \mathrm{pc})$ and that the bar contains the dominant mass of this region. The stars move on elliptical $x_{1}$ and $x_{2}$ orbits, which is apparently also the case for the dominant part of 
Table 2. Parameters of the Adopted Cone Model (shown in Fig. 6) for an inclination of M 82 of $i=75^{\circ}, b=300 \mathrm{pc}$, and $\Delta b=150-200 \mathrm{pc}$.

\begin{tabular}{lccccc}
\hline $\begin{array}{l}\text { Slit position } \\
r \text { [pc] }\end{array}$ & $\begin{array}{c}\text { Opening angle } \\
\Omega\left[^{\circ}\right]\end{array}$ & $\begin{array}{c}\text { Blowout velocity } \\
V_{\mathrm{b}}\left[\mathrm{km} \mathrm{s}^{-1}\right]\end{array}$ & $\begin{array}{c}\text { Cone tilt } \\
\Theta\left[{ }^{\circ}\right]\end{array}$ & $\begin{array}{c}\text { Vel. shift } \\
{\left[\mathrm{km} \mathrm{s}^{-1}\right]}\end{array}$ & $\begin{array}{c}\text { Velocity increase } \\
a\left[\mathrm{~km} \mathrm{~s}^{-1} \mathrm{kpc}^{-1}\right]\end{array}$ \\
\hline SE Cone & & & & & \\
Center: 0 & 25 & 400 & 3 & 0 & 300 \\
West: -300 & 25 & 400 & 0 & 0 & 300 \\
East: +300 & 25 & 400 & 6 & 0 & 300 \\
\hline NW Cone & 20 & 300 & 0 & -100 & 800 \\
Center: 0 & 25 & 350 & 0 & -100 & 900 \\
West: -300 & 23 & 350 & 0 & -100 & 500 \\
East: +300 & & & & & \\
\hline \hline Adopted MODEL & 25 & 400 & 3 & 0 & 300 \\
SE cone & 25 & 350 & 0 & $\sim-100$ & 750 \\
NW cone & & & & & \\
\hline
\end{tabular}

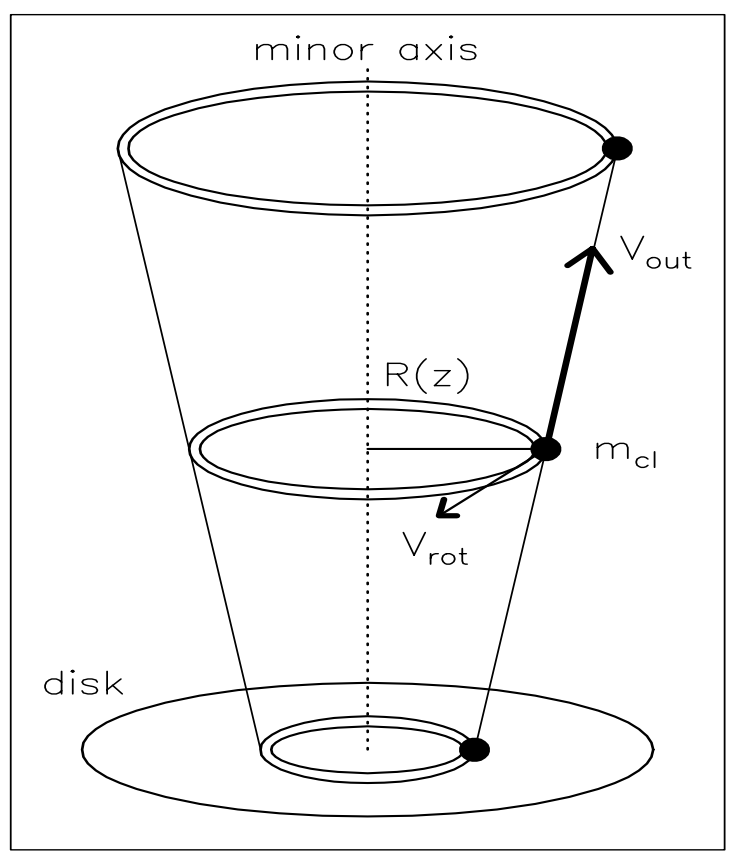

Fig. 5. Momentum conservation of outflow material moving outwards along the cone walls (a tangential element is shown). The arrow indicates the trajectory of the outflowing material.

the (molecular) gas, as evident from the $\mathrm{CO}$ observations by Shen \& Lo (1995) and Neininger et al. (1998). The starburst OB stars, which eventually produce the material of the outflow by supernova explosions, may follow similarly ordered orbits. Because of the low ellipticity of the stellar orbits (0.6-0.9; see Achterman \& Lacy 1995; Wills et al. 2000), we assume that the outflow material moves inside and outside the disk in more or less circular orbits.

\subsection{Radio observations (CO) above the disk}

Information on the rotation of material above and below the disk $(200 \mathrm{pc} \lesssim Z)$ is available from mm-wavelength $\mathrm{CO}$ observations. From the observation by Sofue et al. (1992) it appears that the $\mathrm{CO}$ above and below the disk rotates in the same direction as the disk, and that the rotation of the disk of $v( \pm 300) \approx \pm 100 \mathrm{~km} \mathrm{~s}^{-1}$ at the distance $r \approx b \approx \pm 300 \mathrm{pc}$ from the center (McKeith et al. 1993; Greve et al. 2002) decreases to $V_{\text {rot }}( \pm 300, Z) \approx \pm 50 \mathrm{~km} \mathrm{~s}^{-1}$ at the distance $Z \approx 0.3-0.4 \mathrm{kpc}$; to $V_{\text {rot }}( \pm 300, Z) \approx \pm 20-30 \mathrm{~km} \mathrm{~s}^{-1}$ at the distance $Z \approx 1 \mathrm{kpc}$; and that the $\mathrm{CO}$ and the rotation disappear at the distance $Z \approx 1.5$ kpc. Seaquist \& Clark (their Fig. 5) provide CO rotation curves at different distances above and below the disk, up to a distance of $Z \approx 630 \mathrm{pc}$. These curves confirm that the $\mathrm{CO}$ rotates in the same direction as the disk, and that the rotation decreases with increasing distance from the disk. Globally, the steepest part of the rotation curves is observed between opposite sides at $\theta(z) \lesssim \pm 40^{\prime \prime}(r(z) \lesssim \pm 600 \mathrm{pc})$ distance from the minor axis, for all heights where $\mathrm{CO}$ rotation curves are determined. The $\mathrm{CO}$ rotation curves show slow(er) rotation, or even a reversed rotation, between $40^{\prime \prime} \lesssim \theta \lesssim 60^{\prime \prime}(600 \mathrm{pc} \lesssim r(z) \lesssim$ $900 \mathrm{pc})$ and $-60^{\prime \prime} \lesssim \theta \lesssim-400^{\prime \prime}(-900 \mathrm{pc} \lesssim r(z) \lesssim-600 \mathrm{pc})$ distance from the minor axis. These outer regions are apparently located at the periphery of the visible cones, although this interpretation needs further observational confirmation.

To quantify the decrease of rotation with distance from the disk, we have derived the steepness of the $\mathrm{CO}$ rotation curves $\left[\mathrm{km} \mathrm{s}^{-1} /{ }^{\prime \prime}\right]$ as function of distance from the galactic plane, i.e.

$$
\begin{aligned}
\mathcal{G}(z) & =\left[\boldsymbol{V}_{\mathrm{obs}}\left(r_{1}, z\right)-\boldsymbol{V}_{\mathrm{obs}}\left(r_{2}, z\right)\right] / \Delta r(z) \\
& =\left[\boldsymbol{V}_{\text {rot }}\left(r_{1}, z\right)-\boldsymbol{V}_{\text {rot }}\left(r_{2}, z\right)\right] / \Delta r(z)=\Delta V / \Delta r
\end{aligned}
$$

$\Delta V$ in Eq. (11) is the velocity difference of the $\mathrm{CO}$ emission measured at corresponding opposite positions at the Eastern side $\left[r_{1}(z)\right]$ and Western side $\left[r_{2}(z)\right]$ of the minor axis $\left(\left|r_{1}\right| \approx\right.$ $\left.\left|r_{2}\right|\right)$, separated by the distance $\Delta r=\left|r_{1}\right|+\left|r_{2}\right|$. The values $\mathcal{G}$ are normalized to the value $\mathcal{G}(0)$ measured in the galactic plane, i.e.

$\gamma=\mathcal{G}(z) / \mathcal{G}(0)$.

As evident from Eq. (11), the parameter $\gamma$ eliminates the outflow component $\boldsymbol{V}_{\text {out }}$ but contains the factor $\cos \varphi(r, z)$ which relates the positions $\left(r_{1}, z\right)$ and $\left(r_{2}, z\right)$ to the cone axis and the projection of the velocity of rotation to this direction of the line-of-sight. Figure 7 shows the values $\gamma / \cos \varphi$ as function of distance from the galactic plane. In this figure the factor $\cos \varphi$, based on the cone parameters of CO material (Table 1), has been considered as far as possible. The values $\gamma$ derived from the $p-v$ diagrams of CO measured by WWS- 02 at \pm 330 pc distance from the disk are also shown in Fig. 7. The data suggests 


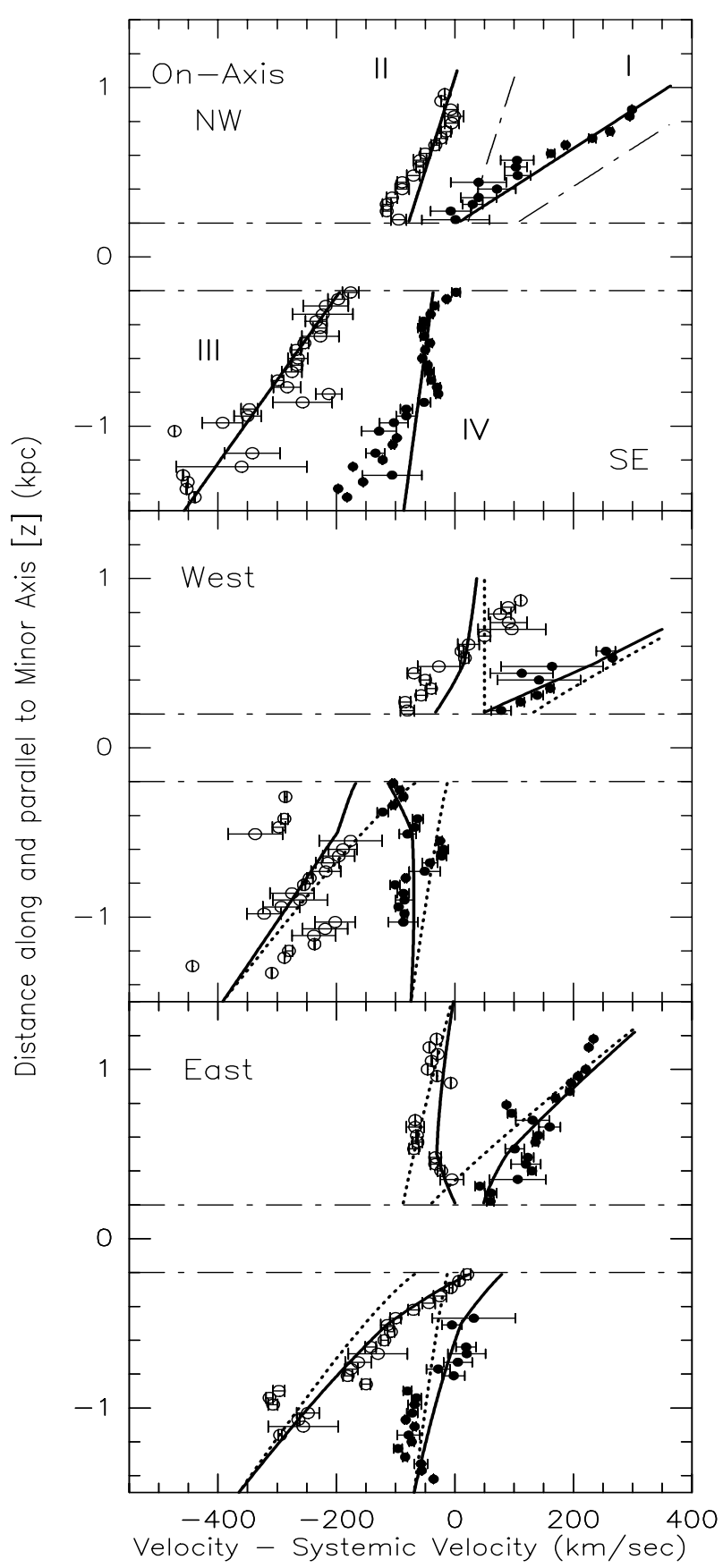

Fig. 6. Illustration of the agreement between the observation and the adopted cone model (Table 2). The displayed data are the averages of the velocities determined from the $\mathrm{H}_{\alpha}$, [NII] and [SII] lines. Open circles: front sides of the cone; dots: rear sides. The solid lines represent the calulated velocity branches when rotation around the minor axis with angular momentum conservation $\boldsymbol{L}_{\mathrm{vis}}$ is applied. Note that in the on-axis observation (top) the rotation velocity is perpendicular to the line-of-sight and hence not seen. The dashed lines show the calculated velocity branches without rotation around the minor axis. Observation West at $\theta=-20^{\prime \prime}$ from the center, observation East at $\theta=20^{\prime \prime}$ from the center. The dashed-dotted line in the on-axis observation (top) is a calculation without the velocity shift of $-100 \mathrm{~km} \mathrm{~s}^{-1}$ of the cone at the NW side.

a faster decrease of $\mathrm{CO}$ rotation at the SE side of the disk, as pointed out by $\mathrm{SC}-01$.

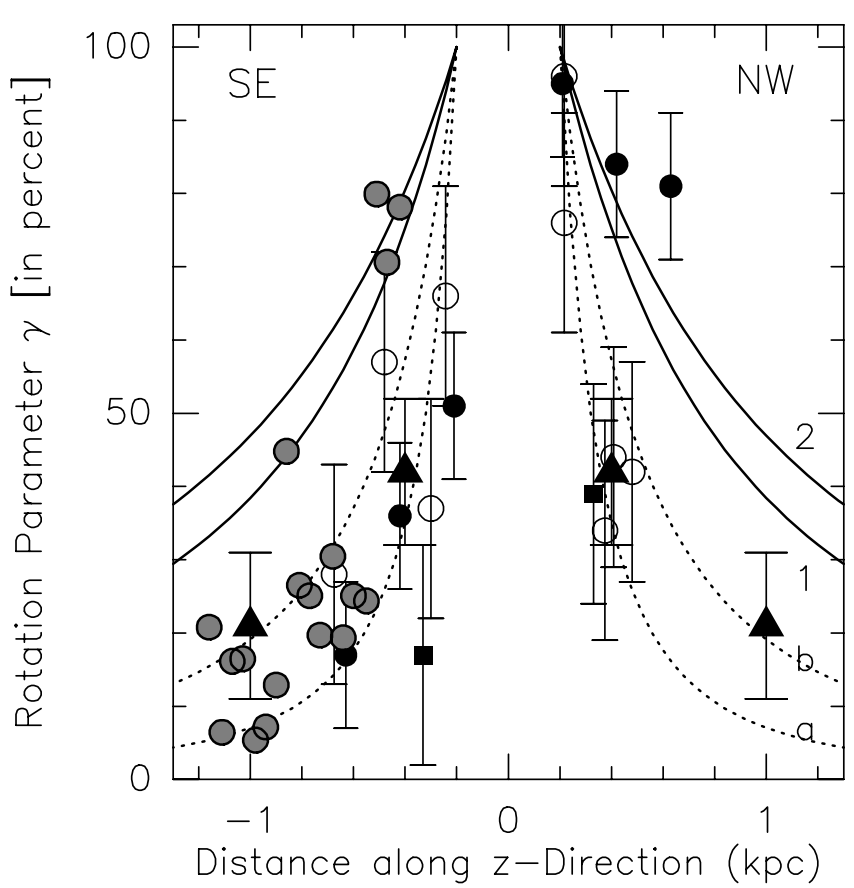

Fig. 7. Values of the rotation parameter $\gamma$ (Eqs. (11) and (12)) above (NW) and below (SE) the disk as derived from observations of CO material and visible cone wall material. We assume that the disk rotates homogeneously within $|z| \lesssim \pm H \approx \pm 200$ pc. Radio data based on the CO observations by Sofue et al. (1992) and Loiseau et al. (1990): triangles; based on CO observations by Seaquist \& Clark (2001): dots; based on CO observations by Walter et al. (2002): squares. Optical data based on $\mathrm{H} \alpha,[\mathrm{NII}]$ and [SII] slit spectra parallel to the major axis by Heckathorn (1972): open circles; this paper: shaded circles. The lines represent the parameter $\gamma$ calculated for outflow with angular momentum conservation $\boldsymbol{L}_{\mathrm{vis}}$ of cone wall gas with $\Omega=25^{\circ}$ and $b=$ $300 \mathrm{pc}$ (1) and $b=400 \mathrm{pc}$ (2) as appropriate for visible material. The dashed lines refer in a similar way to angular momentum conservation $\boldsymbol{L}_{\mathrm{do}}$ of CO cone wall gas with $\Omega=50^{\circ}$ and $b=300 \mathrm{pc}$ (a) and $\Omega=90^{\circ}$ and $b=300 \mathrm{pc}(\mathrm{b})$, as suggested by Seaquist \& Clark (2001) and Walter et al. (2002).

\subsection{Optical observations (filaments) above the disk}

The spectra of visible cone wall material taken by BT-88, HAM-90 and SBH-98 parallel to the major axis well below the disk $(-900 \mathrm{pc} \lesssim z \lesssim-600 \mathrm{pc})$ do not allow a quantitative estimate of rotation around the minor axis, although BT-88 and SBH-98 mention that rotation is seen. The spectra taken by Heckathorn (1972) parallel to the major axis to a distance $Z \leq 675 \mathrm{pc}$ above and below the disk have sloped tracks in the corresponding $p-v$ diagrams (his Figs. 2-17) and by this provide evidence of rotation of the visible cone wall material. As to be expected, the visible cone wall material rotates in the same direction as the disk, and the rotation decreases with increasing distance from the disk. As seen in Heckathorn's Fig. 18, the optical observations $\left(\mathrm{H}_{\alpha},[\mathrm{NII}]\right.$, [SII]) produce a less regular velocity pattern than the $\mathrm{CO}$ observations, and it is impossible to find data at corresponding opposite sides of the minor axis $\left(r_{1}, r_{2}\right)$ from which the parameter $\Delta V / \Delta r$ can be determined. The values $\gamma=(\Delta V / \Delta r) / \mathcal{G}(0)$ obtained from Heckathorn's observation for irregular sections parallel to the major axis are 


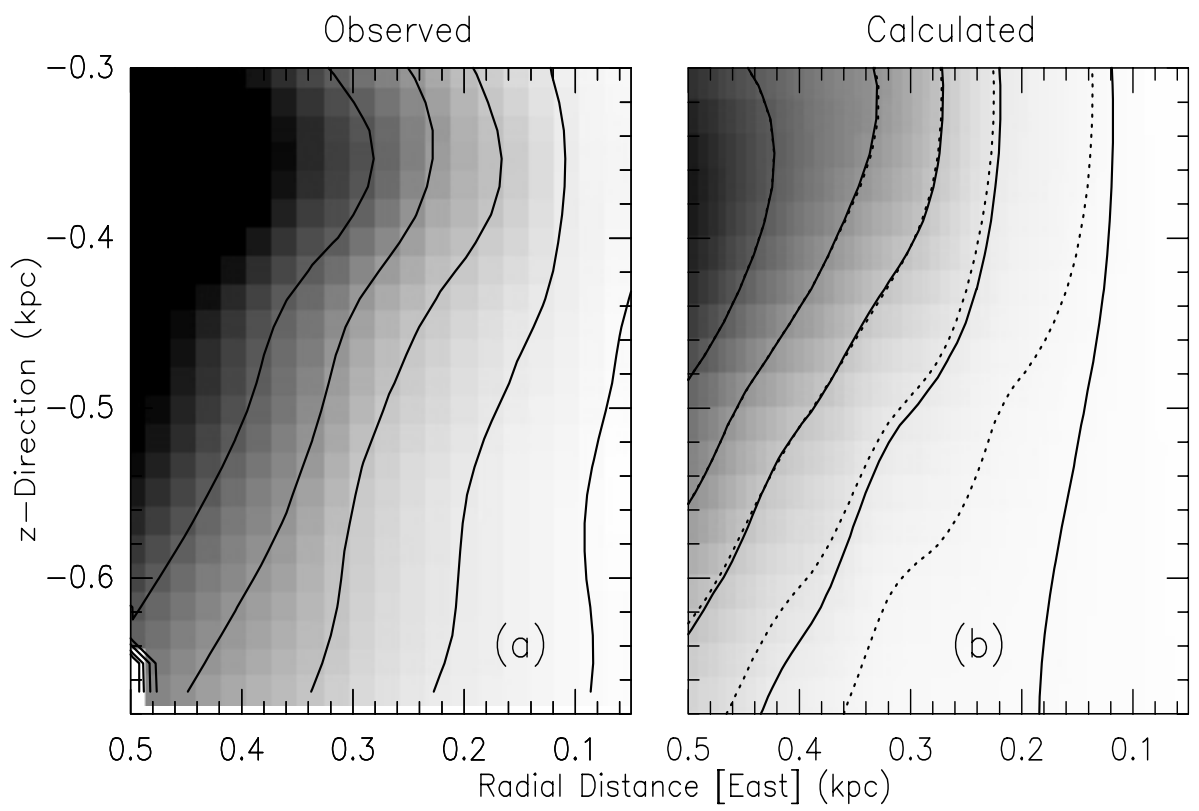

Fig. 8. Momentum conserved rotation of the eastern part of the visible outflow material at the SE side of the disk; plotted as grayscale and lines of values $\boldsymbol{L}=\boldsymbol{V}_{\mathrm{obs}} \times r(z)=$ constant $=5,15,30,45,60 \mathrm{kpc} \times \mathrm{km} \mathrm{s}^{-1}$ (from the minor axis to the outside). a) based on optical line observations by Heckathorn (1972) and this paper; b) calculated from the adopted model of Table 2 . The solid lines show the calculated values $\boldsymbol{V}_{\text {obs }} \times r(z)=$ constant; the dashed lines show the calculated values $\boldsymbol{V}_{\text {rot }} \times r(z)=$ constant, in which the outflow velocity $\boldsymbol{V}_{\text {out }}$ has been removed.

shown in Fig. 7. This figure contains also the values $\gamma$ extracted from our observation at $\pm 20^{\prime \prime}\left(r_{1}, r_{2}\right)$ from the center and the front side (branch III) of the SE cone. The optical emission of the NW cone is too irregular to provide usefull data.

\subsection{Rotation observed at the cone edges}

If we assume that we observe in Fig. 2 at the SE side of the disk the edge of the outflow cone (no velocity splitting), then the velocity difference between the Eastern (red-shifted) and Western (blue-shifted) branch is $\sim 2$ times the rotation velocity of the cone wall material. Taking the straight lines inserted in Fig. 2 as a first approximation of the outflow, the rotation velocity is $\boldsymbol{V}_{\text {rot }}(600, z) \approx 1 / 2 \times(100-150)=50-75 \mathrm{~km} \mathrm{~s}^{-1}$.

\section{Model calculations using rotation}

Using the model of Table 2, the synthetic profiles of the branches I-IV inserted in Fig. 6 show that rotation of the visible cone wall material is required to obtain an acceptable interpretation of the data. The heavy lines in this figure include angular momentum conserved rotation of the cone wall material $\left(\boldsymbol{L}_{\mathrm{vis}}\right)$. The dashed lines are the branches I-IV calculated without rotation, i.e. $V_{\text {rot }}(r, z)=0$ at all distances $z$. This gives a poorer fit to the data.

We have inserted in Fig. 7 the calculated parameter $\gamma$ (Eqs. (11) and (12)) of visible material moving with conserved angular momentum $\boldsymbol{L}_{\mathrm{vis}}$ on a narrow cone $\left(\Omega=25^{\circ}\right)$ as well as the calculated parameter $\gamma$ of dragged-out material moving with conserved angular momentum $\boldsymbol{L}_{\mathrm{do}}$ on a wider cone $\left(\Omega=50^{\circ}\right.$ and $\left.90^{\circ}\right)$. The data shown in Fig. 7 provide evidence of rotation, but unfortunately no clear distinction between visible material on a narrow cone and $\mathrm{CO}$ material on a wider cone.
The figure suggests, to some extent, that the visible material of the SE cone follows first the rotation of the narrow visible cone, but diverts into a wider cone at greater distances from the disk. The large scatter of the data is probably due to the fact that in the construction of Fig. 7 we have used measurements at opposite sides of the minor axis in order to eliminate the outflow velocity $V_{i} \sin \varphi$. These opposite positions are separated by several $100 \mathrm{pc}$ or even a kpc; the irregularities which exists in the outflow over such large distances may produce the large scatter.

As known from Heckathorn's (1972, his Fig. 18) investigation, the outflow in the Eastern quadrant at the SE side of the disk shows the most regular velocity pattern. Using Heckathorn's observation and the data of this publication, we derived for this section of the SE outflow the diagram of constant values $\boldsymbol{V}_{\text {obs }} \times r(z)$ (in which the outflow velocity $V_{i} \sin (\varphi)$ is not eliminated). As displayed in Fig. 8, the contours of constant momentum calculated for the adopted model (Table 2) and momentum conservation $\boldsymbol{L}_{\mathrm{vis}}$ agree with the contours derived from the observations. This figure illustrates the existence of momentum conserved rotation of the visible outflow material.

\section{Summary}

We summarize the result of this investigation and comment on the collimation of the outflow, the escape of the outflow material, the dissipation in the outflow of angular momentum, and the NW-SE asymmetry of the outflow.

\subsection{Results}

(1) The outflow of the visible material occurs along the walls of cones, on both sides of the disk. The parameters of the visible outflow cones are summarized in Table 2. This is an idealized, 
though physically acceptable picture of the visible cone walls despite evidence of an irregular geometry, velocity, and density.

(2) The outflow builds up inside the central $\sim 600-1000 \mathrm{pc}$ of the disk and breaks out of the disk at the height of $\sim \pm 200 \mathrm{pc}$ on the SE and NW side. Our observation detects the visible outflow at $\pm 300 \mathrm{pc}$ and $\pm 600 \mathrm{pc}$ radial distance from the center so that the base over which the visible outflow extends is of the order of $B \approx 600-1200 \mathrm{pc}$ diameter. This is larger than the base of 600-800 pc diameter considered so far.

(3) At the height of $\sim \pm 200 \mathrm{pc}$ above and below the disk exists a sharp discontinuity ( $\sim 50 \mathrm{pc}$ extent) of the outflow; below this height the outflow builds up, above this height the outflow appears to be cone-like with double-split emission lines, on the SE and NW side of the disk. At the discontinuity the de-projected blowout velocity is $V_{\mathrm{b}} \approx 350-400 \mathrm{~km} \mathrm{~s}^{-1}$, on both sides of the disk. The discontinuity is probably the location of the sonic surface beyond which the outflow moves at super-sonic speed (Heckman et al. 1990). This view is supported by the fact that the sound velocity in the tenuous gas is 200-300 $\mathrm{km} \mathrm{s}^{-1}$ (Tomisaka \& Bregman 1983; Strickland \& Stevens 2000). The height of the discontinuity is similar to the vertical distribution of the stars ( $K$ image of $\sim \pm 10^{\prime \prime}= \pm 150 \mathrm{pc}$ extent above and below the plane (Telesco et al. 1991; Larkin et al. 1994)).

(4) The visible SE and NW cones have small opening angles of $\Omega_{\mathrm{SE}} \approx \Omega_{\mathrm{NW}} \approx 20-30^{\circ}$. The shape of these cones is jetlike, in the terminology of Suchkov et al. (1996), and predicted in model calculations assuming a thick disk. The spectroscopic observations show that the visible emission follows the cone walls in a nearly continuous way, with little interruptions and breakout, which gives the impression of a nearly continuous outflow from the disk.

(5) The double velocity-split visible emission lines originate in the walls of the cone-like outflow on the SE and NW side of the disk. The absence, to a large extent, of emission between the velocity branches I-IV (Fig. 3) is evidence of a finite thickness of the cone walls, estimated to be of the order of $\Delta b \approx 150-250$ pc. A similar cone wall thickness is seen in the outflow of NGC 253, which has an opening angle of $\Omega \approx 50^{\circ}$ and a base of $B \approx 600$ pc diameter (Schulz \& Wegner 1992). The absence of velocity splitting, at least of the SE cone, when investigated at $\sim \pm 600 \mathrm{pc}$ radial distance (Fig. 2), indicates that in this region the edge of the visible cone is located.

(6) The outflow velocity of the visible cone wall material increases more or less linearly along the cone walls, at least up to the distance of $\sim 1 \mathrm{kpc}$. The velocity increase is $a_{\mathrm{SE}} \approx 300-400 \mathrm{~km} \mathrm{~s}^{-1} \mathrm{kpc}^{-1}$ at the SE side of the disk and $a_{\mathrm{NW}} \approx 500-800 \mathrm{~km} \mathrm{~s}^{-1} \mathrm{kpc}^{-1}$ at the NW side of the disk. The small inclinations of the velocity branches I-IV above and below $\sim 1 \mathrm{kpc}$ distance from the disk suggests that the cone wall material continues to flow out at a more or less constant velocity (Fig. 1). A similar velocity feature is probably seen in the off-center spectrogram published by Devine \& Bally (1999).

(7) The visible cone wall material rotates in the direction of the galactic disk and with decreasing velocity at greater distances above and below the galactic plane. The rotation seems to conserve angular momentum, as also indicated by the CO observations of Seaquist \& Clark (2001) and
Walter et al. (2002). The rotation velocity is small and of the order of $50 \mathrm{~km} \mathrm{~s}^{-1}$ at the height of $\sim 0.5 \mathrm{kpc}$. A complete rotation of the cone wall material at $\sim 0.5 \mathrm{kpc}$ distance from the disk requires $\sim 10 \mathrm{Myr}$. If we assume that the lifetime of the visible clumps and filaments is small, then the visible outflow material is seen as straight-line streamers rather than helicaltype streamers. The rotation of the outflowing material entrains angular momentum. The entrained and eventually dissipated angular momentum is a small fraction of the angular momentum of the central part of the disk.

(8) Dust continuum observations (Alton et al. 1999) and molecular line (CO) observations by Seaquist \& Clark (2001) and Walter et al. (2002) indicate that the outflow drags cool material out of the disk. The observations show that the outflow of visible cone wall material occurs within significantly narrower cones $\left(\Omega \approx 20-30^{\circ}\right)$ than the outflow of dragged-out material $\left(\Omega \approx 40-90^{\circ}\right)$. The cones of dragged-out $\mathrm{CO}$ material are (presently) only detected to a height of $\sim 650 \mathrm{pc}$ (Seaquist $\&$ Clark 2001), and apparently only on the SE side (Walter et al. 2002), while CO material itself is seen at larger distances from the disk $(Z \lesssim 1 \mathrm{kpc})$.

\subsection{Collimation of the outflow}

We obtain from the earlier and present optical observations the picture of a highly collimated jet-like outflow (in the terminology of Suchkov et al. 1996) of M 82's visible material. This collimation is assumed to be due to the molecular ring of $\sim 600 \mathrm{pc}$ inner diameter $(B)$, of approximately $300 \mathrm{pc}$ radial extent $(\Delta r)$, and of approximately $\pm 150 \mathrm{pc}$ thickness in $z$-direction $(\Delta z)$ (Neininger et al. 1998; Mao et al. 2000; Weiß et al. 2001). The collimation of M 82's outflow may be explained by the favourable "confinement" ratio $R_{\mathrm{c}}=\Delta z / B \approx 1 / 4$ and the relatively high local "compactness" (i.e. high filling factor) of the molecular ring and dust material. This is well seen in the $\mathrm{H}_{\alpha}$ Fabry-Perot image of the outflow published by SBH-98 (their Fig. 9) where the Eastern side of the SE outflow is sharply confined (collimated) by a prominent dust lane which extends nearly perpendicular out of the disk.

Strickland \& Stevens (2000) discuss the collimation efficiency of outflows, in particular of the outflow in M 82, and conclude from hydrodynamic calculations that a starburst in a thin disk produces an outflow with wide cone angle $\left(50-60^{\circ} \lesssim\right.$ $\Omega$ ), a starburst in a thick disk an outflow with narrow cone angle $\left(\Omega \lesssim 40^{\circ}\right)$. We emphasize that the present investigation confirms the highly collimated visible outflow but also that the base of the outflow extends over $B \approx 600-1200 \mathrm{pc}\left(\theta= \pm 20^{\prime \prime}\right.$ of Fig. $1 ; \theta= \pm 40^{\prime \prime}$ of Fig. 2). This fact may solve part of the discrepancy that for M 82 the hydrodynamic model calculations (Tomisaka \& Bregman 1993; Suchkov et al. 1994; Strickland \& Stevens 2000) predict a base of $B \approx 1$ kpc diameter, or even larger. This is significantly larger than the until now adopted base of the visible outflow of $B \approx 600 \mathrm{pc}$, assumed to be comparable in size to the inner diameter of the molecular ring. The presented observations indicate that the base of the visible outflow is as large as the outer diameter of the molecular ring (Table 1). However, we cannot exclude the possibility 
that we observe at this large radial distance some ionized material which is dragged-out of the disk.

\subsection{Escape of the outflow}

The presented observation shows that at $\sim 0.5 \mathrm{kpc}\left(Z_{\mathrm{o}}\right)$ above and below the disk the visible cone wall material moves outward along the cone walls at the true velocity of $500-800 \mathrm{~km} \mathrm{~s}^{-1}$. This velocity is larger than the escape velocity from the disk of $v_{\mathrm{esc}} \approx \sqrt{2 \mathrm{GM} / Z_{\mathrm{o}}} \approx 300 \mathrm{~km} \mathrm{~s}^{-1}$, derived for the stellar mass of $M \approx(2-4) \times 10^{9} M_{\odot}$ concentrated within $\sim \pm 1000 \mathrm{pc}$ radial distance from the center (for the mass of M 82 see Götz et al. 1990; Förster Schreiber et al. 2001; Greve et al. 2002). Most of the visible cone wall material, and probably a major part of the inner tenuous gas, is therefore eventually dispersed into interglactic space. The recent observations (Seaquist \& Clark 2001; Walter et al. 2002) show that the $\mathrm{CO}$ material flows out at a significantly smaller velocity than of the visible material, i.e. $v_{\mathrm{CO}} \approx 250 \mathrm{~km} \mathrm{~s}^{-1} \lesssim 1 / 2 v_{\text {vis }} \approx$ $400 \mathrm{~km} \mathrm{~s}^{-1}$. The velocity $v_{\mathrm{CO}}$ is probably not exceeding the escape velocity so that the major part of the dragged-out material may eventually fall back into the disk at a large radial distance, like in a fountain. Dust at a large distance from the disk showing this fountain-like behaviour is probably seen in the observation by Ichikawa et al. (1994) and Alton et al. (1999). In this picture it is understandable that the cone angle of the fan-like (in the terminology of Suchkov et al. 1996) dragged-out material is significantly larger than the cone angle of the visible cone wall material, i.e. $\Omega_{\mathrm{vis}} \approx 20-30^{\circ}<\Omega_{\mathrm{CO}} \approx 50^{\circ}$, or even larger. The dragged-out material is probably located mostly at the periphery of the visible cones.

\subsection{Angular momentum dissipation}

M 82's rotating and partially escaping outflow dissipates matter and angular momentum which, however, is only a small amount of the angular momentum of the material of the disk and hence of little consequence for the kinematic evolution of the galaxy. This can be seen from the comparison of the angular momentum $\boldsymbol{L}_{\mathrm{fl}}$ dissipated in the outflow during the time of the recent starburst of $T_{\mathrm{stb}} \approx 10^{7}$ years (O'Connell et al. 1995) with the angular momentum $\boldsymbol{L}_{\text {disk }}$ of the material inside the disk of $2 r \approx 2 \times 1000 \mathrm{pc}$ diameter. In order to calculate $\boldsymbol{L}_{\mathrm{fl}}$ we assume that the material inside the cones and the cone walls was and is the major part of the material ejected in the supernova explosions of the heavy stars created in the starburst. We assume that this outflowing material rotates, as known to be the case of the molecular material and shown above to be the case of the visible material. The ejected material eventually escapes. The amount of angular momentum $\boldsymbol{L}_{\mathrm{fl}}$ dissipated by this material during the starburst time is

$$
\begin{aligned}
\boldsymbol{L}_{\mathrm{fl}} \approx M<r><V_{\mathrm{rot}}> & \lessgtr 2 \times 10^{8} M_{\odot} \times 500 \mathrm{pc} \times 50 \mathrm{~km} \mathrm{~s}^{-1} \\
& \lessgtr 5 \times 10^{12} M_{\odot} \mathrm{pckm} \mathrm{s}^{-1} .
\end{aligned}
$$

The mass $M$ in relation (13) is derived under the assumption that the star formation rate $S F R \approx(5-10) \times M_{\odot} \mathrm{yr}^{-1}$ (O'Connell et al. 1995) has persisted throughout the starburst time, and that the heavier stars of some $M_{\mathrm{stb}} \approx 1 / 2 \times S F R \times T_{\text {stb }} \approx$ $(5-10) \times 10^{7} M_{\odot}$ total mass have ended in supernova explosions and that their material has escaped in the outflow. A somewhat lower value is obtained if we assume a constant supernova rate $S N R \approx 0.1 \mathrm{SN} \mathrm{yr}^{-1}$ (Kronberg \& Sramek 1985; Muxlow et al. 1994) throughout the starburst time and if the exploded stars had an average mass of $\left\langle m>\approx 10 M_{\odot}\right.$. Under these assumptions $M_{\mathrm{stb}} \approx S N R \times<m>\times T_{\mathrm{stb}} \approx 10^{7} M_{\odot}$. In addition, the outflow drags material out of the disk and this material transports angular momentum which is partially dissipated. The mass of the dragged-out material $\left(\mathrm{H}_{2}\right)$, presently residing in the halo, is estimated to be $\sim 5 \times 10^{8} M_{\odot}$ (Seaquist \& Clark 2001). However, only a fraction of the dragged-out material may escape while the major part falls back into the halo. If we assume the fraction of the escaping mass to be $M_{\mathrm{do}} \approx(1 / 10-1 / 3) \times 5 \times 10^{8} \approx(0.5-1) \times 10^{8} M_{\odot}$, we obtain for the mass $M$ in relation (13)

$M \lessgtr M_{\mathrm{stb}}+M_{\mathrm{do}} \approx 2 \times 10^{8} M_{\odot}$.

In relation (13) the average distance of the outflow material from the minor axis is assumed to be $\langle r\rangle \approx 500 \mathrm{pc}$, the average rotation velocity $<V_{\text {rot }}>\approx 50 \mathrm{~km} \mathrm{~s}^{-1}$.

On the other hand, the angular momentum $\boldsymbol{L}_{\text {disk }}$ is obtained when using in the relation

$\boldsymbol{L}=\int m(r) r v(r) \mathrm{d} r$

the mass distribution $m(r)$ derived by Götz et al. (1990; see also Förster Schreiber et al. 2001) and the galactic rotation $v(r)$ derived by Greve et al. (2002; see also McKeith et al. 1993). In this way we obtain

$\boldsymbol{L}_{\mathrm{disk}}=2 \times 10^{14} M_{\odot} \mathrm{pc} \mathrm{km} \mathrm{s}^{-1}$.

Allowing for a large uncertainty in this estimate, the ratio $\boldsymbol{L}_{\mathrm{f}} / \boldsymbol{L}_{\mathrm{disk}} \approx 1 / 50-1 / 100$ indicates that during the $\sim 10^{7}$ years of the starburst only a small amount of angular momentum of the central part of the disk has been dissipated in the outflow.

\subsection{Asymmetry of the outflow}

There exists in the optical and the CO observations of M 82 an asymmetry between the outflow at the NW and SE side of the disk. This asymmetry is not due to the fact that on the NW side the line-of-sight passes through a more extended layer of visual extinction because of M 82's inclination in this direction ${ }^{3}$. The asymmetry exists even at larger distances from the disk since wide-field deep optical images (for instance Lynds \& Sandage 1963; Devine \& Bally 1999) show that the NW outflow is much wider than the SE outflow, and that the axes of the cones are not co-linear (for instance Deharveng \& Pellet 1970). In addition, our observation seems to indicate that the visible NW cone has a significant velocity component in the direction of the observer and also a significantly larger acceleration along the cone walls. Finally, the $\mathrm{CO}$ distribution and the $\mathrm{CO}$ outflow

\footnotetext{
3 Actually, Figs. 1 and 10 in Lynds \& Sandage (1963) give the impression that the visual extinction is larger at the southern side of the disk.
} 
kinematics is significantly different at the SE and NW side of the disk (Seaquist \& Clark 2001; Walter et al. 2002) which, however, in this case may be due to an irregular distribution of material produced in the tidal interaction between M 81 and M 82 (Cottrell 1986; Yun et al. 1994). It is important to perform a closer study of the origin of this asymmetry and of a possible build-up of this asymmetry inside the disk.

Acknowledgements. Part of the observations at RGO, La Palma (Spain), were made in service time. I thank the observatory for efficient assistence. A. Weiß made a copy of the publication WWS-02 available prior to publication. I appreciated the discussion with D. Downes (IRAM). I thank the referee for his comments, his clarification of momentum conservation, and his advice to throw out the straw while keeping the oats.

\section{References}

Achtermann, J. M., \& Lacy, J. H. 1995, ApJ, 439, 163

Alton, P. B., Davies, J. I., \& Bianchi, S. 1999, A\&A, 343, 51

Amirkhanyan, A. S., Gagen-Torn, V. A., \& Reshetnikov, V. P. 1982, Astrofizica, 18, 31

Amirkhanyan, A. S., Gagen-Torn, V. A., \& Reshetnikov, V. P. 1985, Astrofizica, 22, 239

Axon, D. J., \& Taylor, K. 1978, Nature, 274, 37

Bland, J., \& Tully, R. B. 1988, Nature, 334, 43 (BT-88)

Bregman, J. N., Schulman, E., \& Tomisaka, K. 1995, ApJ, 439, 155

Burbidge, E. M., Burbidge, G. R., \& Rubin, V. C. 1964, ApJ, 140, 942

Chevalier, R. A., \& Clegg, A. W. 1985, Nature, 317, 44

Cottrell, G. A. 1977, MNRAS, 178, 577

Deharveng, J. M., \& Pellet, A. 1970, A\&A, 9, 181

Devine, D., \& Bally, J. 1999, ApJ, 510, 197

Dietz, R. D., Smith, J., Hackwell, J. A., Gehrz, R. D., \& Grasdalen, G. L. 1986, AJ, 91, 758

Freedman, W. L., Hughes, S. M., Madore, B. F., et al. 1994, ApJ, 427, 628

Förster Schreiber, N. M., Genzel, R., Lutz, D., et al. 2001, ApJ, 552, 544

Götz, M., McKeith, C. D., Downes, D., \& Greve, A. 1990, A\&A, 240, 52

Greve, A., Wills, K. A., Neininger, N., \& Pedlar, A. 2002, A\&A, 383, 56

Hartquist, T. W., Dyson, J. E., \& Williams, R. J. R. 1997, ApJ, 482, 182

Heckathorn, H. M. 1972, ApJ, 173, 501

Heckman, T. M., Armus, L., \& Miley, G. K. 1990, ApJS, 74, 833 (HAM-90)

Ichikawa, T., van Driel, W., Aoki, T., et al. 1994, ApJ, 433, 645

Kronberg, P. P., \& Sramek, R. A. 1985, Science, 277, 28
Larkin, J. E., Graham, J. R., Matthews, K., et al. 1994, ApJ, 420, 159

Lehnert, M. D., Heckman, T. M., \& Weaver, K. A. 1999, ApJ, 523, 575

Loiseau, N., Nakai, N., Sofue, Y., Wielebinski, R., \& Klein, U. 1990, A\&A, 228, 331

Lynds, C. R., \& Sandage, A. R. 1963, ApJ, 137, 1005

Mao, R. Q., Henkel, C., Schulz, A., et al. 2000, A\&A, 358, 433

McKeith, C. D., Castles, J., Greve, A., \& Downes, D. 1993, A\&A, 272, 98

McKeith, C. D., Greve, A., Downes, D., \& Prada, F. 1995, A\&A, 293, 703 (M-95)

Muxlow, T. W. B., Pedlar, A., Wilkinson, P. W., et al. 1994, MNRAS, 266, 455

Nakai, N., Hayashi, M., Handa, T., Sofue, Y., \& Hasegawa, T. 1987, PASJ, 39, 685

Neininger, N., Guelin, M., Klein, U., Garcia-Burillo, S., \& Wielebinski, R. 1998, A\&A, 339, 737

O'Connell, R. W., Gallagher, J. S. III, Hunter, D. A., \& Colley, W. N. 1995, ApJ, 446, L 10

Ohyama, Y., Taniguchi, Y., Iye, M., et al. 2002, PASJ, 54, 891

Sakai, S., \& Madore, B. F. 1999, ApJ, 526, 599

Scarrott, S. M, Eaton, W., \& Axon, D. J. 1991, MNRAS, 252, 12

Schulz, H., \& Wegner, G. 1992, A\&A, 266, 167

Seaquist, E. R., \& Clark, J. 2001, ApJ, 552, 133 (SC-01)

Shen, J., \& Lo, K. Y. 1995, ApJ, 445, L 99

Shopbell, P. L., \& Bland-Hawthorn, J. 1998, ApJ, 493, 129 (SBH-98)

Sofue, Y., Reuter, H.-P., Krause, M., Wielebinski, R., \& Nakai, N. 1992, ApJ, 395, 126

Suchkov, A. A., Balsara, D. S., Heckman, T. M., \& Leitherer, C. 1994, ApJ, 430, 511

Suchkov, A. A., Berman, V. G., Heckman, T. M., \& Balsara, D. S. 1996, ApJ, 463, 528

Strickland, D. K., Ponman, T. J., \& Stevens, I. R. 1997, A\&A, 320, 378

Strickland, D. K., \& Stevens, I. R. 2000, MNRAS, 314, 511

Telesco, C. M., Campins, H., Joy, M., Dietz, K., \& Decher, R. 1991, ApJ, 369, 135

Tenorio-Tagle, G., \& Muñoz-Tuñon, C. 1997, ApJ, 478, 137

Thuma, G., Neininger, N., Klein, U., \& Wielebinski, R. 2000, A\&A, 358,65

Tomisaka, K., \& Ikeuchi, S. 1988, ApJ, 330, 695

Tomisaka, K., \& Bregman, J. N. 1993, PASJ, 45, 513

Umemura, S., Iki, K., Shibata, K., \& Sofue, Y. 1988, PASJ, 40, 25

Walter, F., Weiß, A., \& Scoville, N. 2002, ApJ, 580, L 21 (WWS-02)

Weiß, A., Neininger, N., Hüttemeister, S., \& Klein, U. 2001, A\&A, 365,571

Wills, K. A., Das, M., Pedlar, A., Muxlow, T. W. B., \& Robinson, T. G. 2000, MNRAS, 316, 33

Yokoo, T., Fukue, J., \& Taniguchi, Y. 1993, PASJ, 45, 687

Yun, M. S., Ho, P. T. P., \& Lo, K. Y. 1994, Nature, 372, 530 